\title{
Modifying the Traditional Analyzing Method of Cylindrical Workpieces' Deep Drawing Process by Using a Novel Loop Material Theory Method
}

\author{
Rongxin Yang1, Zhengwu Lin ${ }^{2 *}$ \\ ${ }^{1}$ Office of Science and Technology, South China University of Technology, Guangzhou, China \\ ${ }^{2}$ Chenghai Seals Plant, Chenghai, China \\ Email:*teakingi@163.com
}

How to cite this paper: Yang, R.X. and Lin, Z.W. (2017) Modifying the Traditional Analyzing Method of Cylindrical Workpieces' Deep Drawing Process by Using a Novel Loop Material Theory Method. Engineering, 9, 351-360.

https://doi.org/10.4236/eng.2017.94019

Received: March 29, 2017

Accepted: April 25, 2017

Published: April 28, 2017

Copyright (C) 2017 by authors and Scientific Research Publishing Inc.

This work is licensed under the Creative

Commons Attribution International

License (CC BY 4.0).

http://creativecommons.org/licenses/by/4.0/

\begin{abstract}
In this paper, the traditional empirical coefficient method and a novel loop material analytical method presented by the authors have been compared and discussed by analyzing several practical cylindrical workpieces' deep drawing projects. Also, some conclusions about how to modify the traditional analyzing method of cylindrical workpieces' deep drawing process could be concluded and the necessity of this modification could be proved.
\end{abstract}

\section{Keywords}

Cylindrical Workpieces, Deep Drawing Process, Loop Materials, Loop Material Plastic Yield Genes, Analytical Model

\section{Introduction}

Technically in traditional way, the deep drawing coefficient (table) is generally taken as an experimental parameter for the analysis of cylindrical workpieces' stamping and deep drawing process. The drawing coefficient can be defined as the ratio of the outer diameter of slab to the diameter of tube after drawing [1]. However, this kind of parameter is not directly correlated with the loop material which will suffer plastic yield in practical application, so it is hard to find out its accurate deformation rules in this way and this inaccurate method will lead readers or engineers to an incorrect comprehension.

In order to solve this problem, a novel loop material theory analytical method, which is established by using 3 loop material parameters (loop material width $b$, plate thickness $t$ and inner diameter of drawing cylindrical workpieces $d$ ), has been proposed by Lin et al. in 2009 [2] [3]. The loop material which will suffer plastic yield during deep drawing process has been taken as an object, then sev- 
eral experiments have been done to establish and verify a mathematical analytical model including the 3 parameters mentioned above.

It is important that the novel loop material theory could improve the accuracy and reasonability of the traditional method of cylindrical workpieces' deep drawing process in a practical way. Several process analysis examples will be taken to analyze and compare these two methods in this paper.

\section{The Basic Plastic Yield Characteristic of Loop Material Formed into Cylinder}

Practically, when rigid-plastic plate forms into cylindrical workpieces by stamping and deep drawing process, only the loop material outside the cylinder bottom will suffer plastic yield, which means the part of cylinder bottom remains unchanged. The result of cylindrical workpieces deep drawing process is usually determined by the extent of plastic deformation of the loop material, so how to obtain the extent of plastic yield is a key point to distinguish the experimental analysis and scientific analysis. However, rare researches have been done to solve this problem.

In the loop material theory, the extent of plastic deformation can be expressed by mathematical terms $(b / d)$ and $\log (b / t)$, which are the basic mathematical characteristics of loop material plastic yield, and these two terms can also be called Loop Material Plastic Yield Genes (LPG).

According to reference [2], taking the deep drawing process of a plate forms into a straight cylinder as an example, the loop material without the bottom part will be drawn into a cylinder with plastic deformation.

As shown in Figure 1, the deep drawing process can be regarded as a process that the basic rectangle loop material (the white area) digest other triangle material (the dark area) (the rectangle material, called basic loop material, can be regarded as numerous tiny rectangle materials which can be easily folded into a cylinder whose height is $b$ ). Also can be seen in Figure 1, the geometry digestible rate of loop material can be analysed geometrically as:

$$
N_{0}=\frac{0.25 \pi\left(D^{2}-d^{2}\right)-0.5 \pi d(D-d)}{0.5 \pi d(D-d)}=\frac{b}{d}
$$

where $D$ is outer diameter and $d$ is inner diameter.

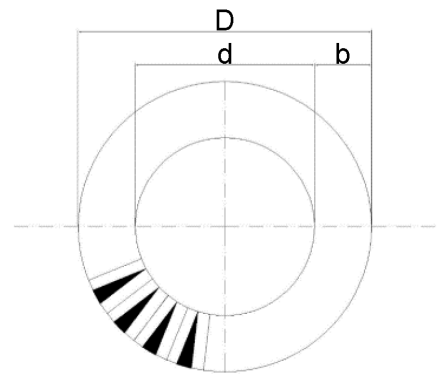

Figure 1. The geometry digestible rate of loop material. 
In addition, because of the influence of cold-work hardening and relative width of loop material $(b / t)$, a relative width coefficient of loop material $\log (b / t)$ should be considered. Hence, the material digestible rate of loop material can be defined as:

$$
N=(b / d) \log (b / t)
$$

Simply, the material digestible rate of loop material can be seen as the product of geometry digestible rate and relative width coefficient. Like the genes in biology, $N$ can be seen as a basic parameter (or can be called as basic characteristic factor of drawing deformation) which can indicate the extent of plastic yield of loop material or can be regarded as a basic characteristic factor in other related process analysis project, so it can also be called as Loop Material Plastic Yield Genes.

In order to simplify the analytical model, $N$ could be used. Moreover, the less magnitude of $N$ means smaller deformation of the workpieces, which means it will be easier to drawing, and vice versa. With the help of this Loop Material Plastic Yield Gene, more process analysis problems such as multiple drawing, suitable drawing load or blank holder force, cylinder blank modification and suitable selection of die fillets can be solved efficiently.

\subsection{The First Ultimate Drawing Problems of Flangeless Cylindrical Workpieces with Blank Holder}

In the traditional way, the blank relative thickness $(T=100 \mathrm{t} / D)$ is generally used to divide the interval value as a criteria, and then the range of ultimate drawing coefficient can be obtained from the related table, for example in Table 1.

Unfortunately, the only thing we can know is "when the die fillet radius $R=8 \sim$ $15 t, m_{1}$ should be the smaller value, and when $R=4 \sim 8 t, m_{1}$ should be the bigger one". However, a judgement formula can be obtained in this novel loop material theory [4]:

$$
N=(b / d) \log (b / t) \leq A J\left(R_{d} / x t\right)^{n}
$$

where $(b / d)$ is the geometry digestible rate of loop material and $(b / t)$ is the relative width of loop material. On the other side of the formula, $A$ is a constant parameter related to the property of material, $J$ is a coefficient parameter related to mould gap, blank holder force, lubrication condition, drawing speed, etc. $\left(R_{d} / x t\right)^{n}$ is the influence coefficient of die fillets, $R_{d}$ is the radius of die fillets, $x$, which is usually equals 8 , is a multiple of $t$ and $n$ is an exponent.

At the initial analysing step, the influence coefficient of die fillets can be assumed to be $\left(R_{d} / x t\right)^{n}=1$. Taking AISI 1008 Steel as an example, assuming that $J=1,\left(R_{d} / x t\right)^{n}=1, A=0.69(A=0.69$ can be obtained by the professional

Table 1. Experimental coefficient $m_{1}$ according to the range of blank relative thickness. 
experiments [4]), the judgement formula can be simplified as:

$$
N=(b / d) \log (b / t) \leq 0.69
$$

As can be seen from Equation (4), the material digestible rate of loop material $N$ will not be larger than $A J\left(R_{d} / x t\right)^{n}$ when the deep drawing process is successful.

By comparison, some conclusions can be concluded. Firstly, significant impacts of the plate thickness and cold-work hardening on deep drawing process are considered in both methods. In traditional way, the ultimate drawing coefficients are the same in the case of equal relative thickness, regardless of the thickness of plate. Similarly, the results will be the same with calculating by Equation (4).

Firstly according to reference [1], $T=2, m_{1}=0.45, T=0.8, m_{1}=0.52$ and $T=$ $0.08, m_{1}=0.63$ can be taken as examples, and $N$ is $0.696,0.682$ and 0.694 , respectively. Secondly, the relative thickness of the whole blank is chosen to be considered in traditional method, but in the proposed novel method, the relative thickness of loop material which has practical plastic deformation is used, and can be defined as $b / t$.

More importantly, in traditional method, the parameters of ultimate drawing coefficient are dependent on the relative thickness of the whole blank (defined as $D(t)$, will be shown as independent variables in a particular equation. In details, this particular equation is a regression equation which can be obtained by the intermediate value from the deep drawing coefficient table (e.g. $m_{1}=0.53$ in case of $T=1.0, m_{1}=0.55$ in case of $\left.T=0.6\right)$, and can be written as:

$$
(b / d) \log (D / t)=0.93 i
$$

Comparing to the results obtained from the deep drawing coefficient table, the curves of regression equation are close to the curves of intermediate value (errors are all less than 0.02), which means the regression equation is effective. As mentioned before, $D$ or $(D / t)$ using as independent variables are incorrect, because the fact that only loop material has the plastic deformation has been ignored [5].

Thirdly, it is hard to know which kind of parameters is better in the traditional method in the practical application. For instance, it is hard to decide which range ( $T<1.5 \sim 1.0$ or $T<1.0 \sim 0.6$ ) should be referred to $m_{1}$ when the relative thickness is 1 exactly. In this novel method, the influence of die fillets on ultimate deep drawing process can be reflected directly by using the influence coefficient of die fillet $\left(R_{d} / x t\right)^{n}$ calculating in the equation.

Fourthly, the calculation of drawing times of multi-stage deep drawing can be obtained conveniently by this judgment formula, which can be defined as:

$$
n= \begin{cases}\left|\frac{N_{t}}{A}\right| & \text { if }\left|\frac{N_{t}}{A}\right| \geq \frac{N_{t}}{A}, \\ \left|\frac{N_{t}}{A}\right|+1 & \text { if }\left|\frac{N_{t}}{A}\right|<\frac{N_{t}}{A}\end{cases}
$$


where $N_{t}$ is the total material digestible rate of loop material (from the original blank to the final cylindrical workpiece).

According to the data of multi-stage deep drawing in existing traditional documents, some comparison and calculation between deep drawing process and the material digestible rate of loop material can be obtained. It can be found that the material digestible rate of loop material during the second-stage deep drawing process is even larger than the one of blank during the first-stage deep drawing process. Moreover, the one of the multi-stage deep drawing process will decrease with the decreasing of die radius after the second-stage deep drawing process, which means $N \leq A J$ in case of $R_{d}=8 t$ and the judgment formula can be used in any stage deep drawing process.

\subsection{Drawing Force Analytical Formula}

In traditional method, the nominal maximum drawing force $P$ can be defined as:

$$
P=3\left(\sigma_{b}+\sigma_{s}\right)\left(D-d-r_{d}\right) t
$$

Actually, it has been found that it is not reasonable in practical application. Hence, a parameter $K_{1}$, which is related to the extent of drawing deformation, can be used efficiently to refer as formula:

$$
F=\pi d t \sigma_{b} K_{1}
$$

However, in loop material theory, it is much more efficient when the formula includes the judgment formula mentioned above. Specifically, at the critical state that drawing force equals to the ultimate tensile stress, the resistance coefficient of loop material of loop material is $(N / A J)^{m}$. And actually, die fillets should be considered, so Equation (8) can be rewritten as:

$$
F=\pi d t \sigma_{b}(N / A J)^{m}(x t / R)^{n m}
$$

Compared to the results which is calculating by using the table in page 258 of reference [1], the analytical results of Equation (9) are surprisingly similar (most errors are less than 0.03), which means $K_{1}$ can be substituted as the resistance coefficient of loop material $(N / A J)^{m}$.

\subsection{Modification Questions about Blank Expansion of Cylindrical Workpieces}

In the traditional method, the expansion blank size will be reduced proportionally according to the particular formula based on the average thinning coefficient or the area change coefficient, but sometimes the size of the blank with large diameter and low height will be reduced to even less than the drawing cylindrical workpieces. As shown in Figure 2, in the novel theory, the Loop Material Plastic Yield Genes $N$ will be involved in this expansion blank modification calculation [6], and an efficient modified reduction formula can be written as:

$$
\Delta_{D}=\Delta_{r}+\Delta_{b}
$$

where $\Delta_{r}$ is the elongation of the material because of the change of the centre line caused by the tiny fillets, and $\Delta_{b}$ is the elongation of loop material caused 


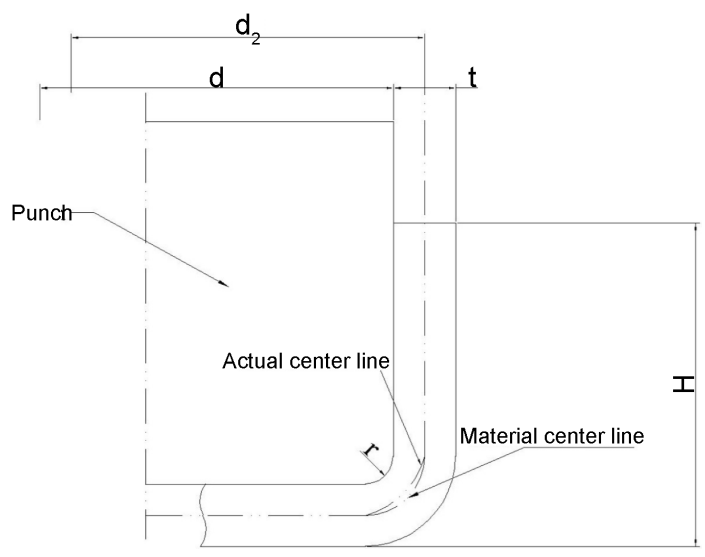

Figure 2. Change of the centre line.

by drawing deformation.

Specifically, $\Delta_{r}$ can be defined as:

$$
\Delta_{r}= \begin{cases}M \pi\left[S-\left(R_{p} / t\right)\right] t & \Delta_{r}>0 \\ 0 & \Delta_{r} \leq 0\end{cases}
$$

where $R_{p}$ is the punch radius, $M$ and $S$ are two constant parameters obtained from experimental data.

Similarly, $\Delta_{b}$ can be defined as:

$$
\Delta_{b}=\sqrt{b}\left[N /\left(R_{d} / t\right)^{1 / 2} \times Z\right] K
$$

where $b$ is the width of loop material, $R$ is the die fillet radius, $Z$ is the ratio of the die side clearance to the plate thickness, $K$ is a comprehensive coefficient (generally equals 1 ) related to pressure condition, lubrication condition, drawing speed, etc. The precise dimension value of blank can be obtained by subtracting the correction quantity from the value of blank calculating by formula.

Equation (10) is suitable for cylindrical workpieces with various dimension, and the errors can be controlled within $0.3 \mathrm{~mm}$ [6], which means it is more precisely than the national standard. Also, the phenomenon, which the size of the blank with large diameter and low height will be reduced to even less than the drawing cylindrical workpieces, will not occur.

\section{The First Ultimate Drawing Problems of Flangeless Cylindrical Workpieces without Blank Holder}

For the narrow loop of the outside loop material which is formed by drawing without blank holder, the ratio of the diameter of the cylinder and the diameter of the blank is used as the drawing coefficient in the traditional way, but it is not reasonable because the bottom part, which is included in the parameter calculation and occupies a large part of the blank area, will keep unchanged during the deep drawing process. In addition, it is hard to know whether the deep drawing process is successful or not. It is not reasonable that when $d / t>75$ ( $d / t$ is the relative cylinder diameter), the width of loop material which will suffer plastic yield remains at 12.5. Also, the influences of particular process conditions (e.g. die fil- 
lets, etc.) are not reflected in traditional method.

In the loop material theory, a standard that judge whether the deep drawing process without blank holder is successful or not is firstly proposed. The rim of formed cylinder becomes irregular can be regarded as failure and the one keeps smooth can be regarded as success.

Considering factors such as die fillets, the diameter of cylinder, etc., a shape coefficient $W$ can be used, and another judgement formula can be defined as:

$$
b^{\prime} \leq C W t\left(R_{d} / y t\right)^{u} \log d
$$

where $b^{\prime}$ is the width of drawing loop material (the one of blank is $\left.b^{\prime}=0.5(D-d)\right), C$ is a constant parameter related to the property of drawing material ( $C$ will be larger if the material is easier to be drawn. e.g. $C=5$ for AISI 1008 Steel). $W$ is a shape coefficient (the value is between $1-1.5$, and should be small if we want a formed cylinder with regular shape rim), $y$ is a multiple of the thickness of loop material (generally equals 10$).\left(R_{d} / y t\right)^{u}$ is a term that reflect the extent of the impact of die fillets and $u$ is an exponent.

Equation (13) indicates that the ultimate width of loop material, drawing without blank holder, will decrease with the diameters of both cylinder and die fillets, based on basic width $C t$.

According to Equation (3), Equation (13) can be rewritten as:

$$
b^{\prime} / t \log d \leq C W\left(R_{d} / y t\right)^{u}
$$

where $b^{\prime} / t \log d \quad$ can reflect the deformation property of the loop material in a suspended state during deep drawing process without blank holder, also can be called the rigid digestible rate of loop material. $b^{\prime} / t$ is the relative width of loop material.

Equation (14) indicates that the rigid digestible rate of loop material, after drawing without blank holder, must be not larger than $C W\left(R_{d} / y t\right)^{u}$.

\section{The Calculation Problems about the Internal Bore Flanging Force}

For the calculation of internal bore flanging force, the formula in traditional method can be defined as:

$$
p=1.1 \pi t \sigma_{s}\left(d-d_{0}\right)
$$

where $\sigma_{s}$ is the yield stress of material, $d$ is the diameter of cylinder after flanging, and $d_{0}$ is the diameter of prefabricate bore.

However, it is also not reasonable because no matter what is the size of cylinder, the flanging forces will be the same if the loop materials' width is equal, and in practice, the fact that the flanging loop material is combined closely with the outer basis should not be ignored.

In addition, it is not correct that the yield stress of material $\sigma_{s}$ has been taken as the parameter because the actual average stress might be greater than $\sigma_{s}$ or even close to the ultimate tensile stress $\sigma_{b}$. The analytical results with Equation (15) are likely to less than the practical one, and the errors will increase with the 
growing of diameters of cylinder, which will have a negative impact.

According to the loop material theory proposed in this paper, the internal bore flanging process must consider two kinds of forces: 1) the bending forces caused by the deformation generated by the connection between flanging loop material and blank matrix, 2) the stretching force generated by the flanging loop material. Hence, the analytical model of the internal bore flanging force, which can be suitable for various cases, is proposed [7]:

$$
F=F_{1}+F_{2}=A \pi d t \sigma_{s}+B \pi t \sigma_{b} b^{m}(b / d)^{n}
$$

where $d$ is the diameter of material that forms to cylinder, $\sigma_{s}$ is the yield stress of material, $b$ is the width of loop material that forms to the straight cylinder, $A$ and $B$ are two calculated distribution coefficients, and $m$ and $n$ are two exponents. $(b / d)$ is the geometric area extension rate of the loop material that forms to the straight cylinder (it will increase if the flanging resistance grows), and $(b / d)^{n}$ is a folding coefficient of $\sigma_{s}$. In addition, $\sigma_{b}(b / d)^{n}$ is the actual average stress of the extension loop material, which will either close to $\sigma_{b}$ or much less than $\sigma_{s}$.

In order to simplify the formula, $b^{m}(b / d)^{n}$ can be simplified as $b / d^{n}$, and $n=$ $1 / 4, A=0.27, B=8$ can be assumed, then the car prefabricated bore flanging force caused by the cylinder head punch can be written as:

$$
F=F_{1}+F_{2}=0.27 \pi d t \sigma_{s}+8 \pi t b \sigma_{b} / d^{n}
$$

where $F_{1}$ is the bending force on the cross section of cylinder and $F_{2}$ is the stretching force on the loop material that forms the cylinder. The analytical results are almost the same as the experimental results, and the errors between them are all less than 4\%, when AISI 1008 Steel has been taken as an example [7].

\section{The Comprehensive Formula}

The deep drawing process of cylinders with notches or lugs is another problem which is different from the deep drawing process of a whole circle loop material or the curve flanging. Besides the target of improving the production efficiency, solving this problem can help to understand deeply about the internal formation during deep drawing process. As shown in Figure 3, in the loop material theory,

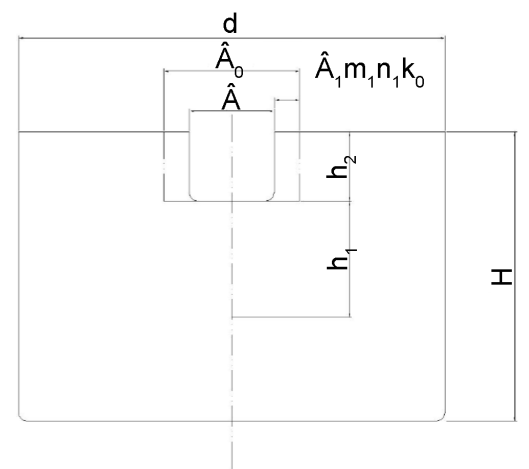

Figure 3. Cylinders with notches or lugs during direct deep drawing process. 
a formula, which can show the relationship between the dimension of the notch of the cylinder after deep drawing and the dimension of the notch of the original blank, has been proposed [8]:

$$
\hat{A}=\hat{A}_{0}-2 \hat{A}_{1} m_{1} n_{1} k_{0}
$$

where $\hat{A}$ is the notch width (or arc length) of the formed cylinder. $\hat{A}_{0}$ is the notch width (or arc length) of the formed cylinder which is drawn from a whole loop material, $m_{1}$ is a coefficient parameter $\left(m_{1}=1 \pm 0.3\right)$ related to blank holder condition, lubrication condition, mould gap, etc. In addition, $n_{1}$ is the tiny notch extrusion coefficient, which can be assumed to be $n_{1}=\left[\hat{A}_{1} \div\left(2 b_{1} h_{1} / F t\right)\right] g \quad\left(n_{1}\right.$ should be required in the case of $\hat{A}_{0} \leq 2 b_{1} h_{1} / F t$, and $g$ is an exponent). $k_{0}$ is impact coefficient of both notch sides, and its value is at a range between 0 and 1 ( $k_{0}$ will decrease when the width of side area decreases).

Specifically, $\hat{A}_{0}$ can be defined as:

$$
\hat{A}_{0}=\pi d \theta / 360
$$

where $\theta$ is the central angle of the notch of the blank.

$\hat{A}_{1}$ is a value of abnormal extrusion on each notch side, and can be defined as:

$$
\hat{A}_{1}=(E / F)\left(b_{1} h_{1} / t\right)
$$

where $E$ and $F$ are two constants and assumed to be $(E / F)=0.078, b_{1}$ is the radial width of loop material outside the blank notch $\left(b_{1} \leq 4.5 t\right.$, otherwise $b_{1}$ can be ignored), $h_{1}$ is the height of the part which below the bottom of the formed cylinder notch $\left(h_{1} \leq 9.5 t\right.$, otherwise $h_{1}$ can be ignored), and $\left(b_{1} h_{1} / t\right)$ can be regarded as a factor value caused by abnormal extrusion on each notch side.

According to Equation (18) mentioned above, it can be noticed that the notch width of the formed cylindrical workpieces is the subtraction that between the notch width (or arc length) of the formed cylinder which is drawn from a whole loop material and the value of abnormal extrusion on each notch side.

Interestingly, it is a deep drawing process of a whole circle loop material problem in the case of $b_{1}=0$, and it is a curve flanging problem in the case of $h_{1}=$ 0, which means Equation (18) is a comprehensive formula that can reflect various kinds of situations. Compared to the experimental result, the errors between analytical results and the practical dimension are all less than $0.5 \mathrm{~mm}$.

$\hat{A}_{1}$ can be regarded as a consequence that caused by the circumferential compressive stress and the radial tensile stress during the deep drawing process.

\section{Conclusion}

It can be concluded that the traditional method has many disadvantages, and the analytical method proposed in this paper can explain clearly and calculate precisely via these formed process examples. The analytical results can be validated by correspondent experiments, and it is analyzed by comparing to the traditional method which uses the deep drawing coefficient (table) in this paper [9] [10].

It can be seen that this proposed loop material theory is more efficient and ef- 
fective than the traditional method since it includes all the factors which should be considered, even though some of formulas seem to be complicated. Moreover, the analytical formula of extension of cylinder can also be deduced by using the relative width of loop material $(b / d)$, which can show the connection between them.

According to the similarity between analytical results and experimental results we have obtained, it can be said that it is an overturning innovation in which the novel loop material theory replaces the traditional method. This new method will not only help to understand the principle inside the deep drawing process, but also help to perfect the computer codes which are used to do the simulation.

\section{References}

[1] Wang, X.P. (1997) Design Information of Stamping. In: 'Information' Is a Form of Shorthand in This Paper, China Machine Press, Beijing.

[2] Lin, Z.W. and Lin, D.H. (2009) Forming Technology Analysis for the Loop Materials of Cylindrical Wall Formed by Plastic Deformation-Introduction to an Invention Patent about Technology Analysis Method for Cylindrical Workpieces Forming. Forging \& Stamping Technology, 34, 57-62.

[3] Lin, Z.W. Technology Analysis Method for Cylindrical Workpieces Forming. Chinese Patent: ZL00107158.0.

[4] Lin, Z.W., Ruan, F. and Zhang, S.J. (1999) The Formula Judging Method on Decision the First Deep-Drawing Limits of Cylindrical Part. Forging \& Stamping Technology, No. 4, 16-18.

[5] Lin, Z.W., Zhang, S.J. and Ruan, F. (1998) An Analysis and Computation Method on Deep-Drawing Blank Correction of Cylindrical Part. Forging and Pressing Academic Annual Meeting Proceedings, Guangzhou, 48-50.

[6] Lin, Z.W. and Lin, D.H. (2002) The Formula Judging Method of the First Time Deep-Drawing Limits of Cylindrical Part without Blank Holder. Forging and Pressing Academic Annual Meeting Proceedings, Guangzhou, 44-47.

[7] Lin, Z.W. (2006) Discussion on Calculation Formula of Edge-Turning Force of Interior Holes. Forging and Pressing Academic Annual Meeting Proceedings, 144-147.

[8] Lin, Z.W. and Cai, R.B. (1997) Discussion on the Process of Barrel Parts with U-Shape Notch. Automobile Technology, No. 8, 39-42, 46.

[9] Lin, Z.W. and Lin, D.H. (2001) The Formula Judging Method of Edge-Turning Limit of Interior Holes. Mechanical \& Electrical Engineering Technology, No. 1, 30-32.

[10] Zheng, L.J. and Xu, H.J. (2014) Reverse Deduction of Cylinder Expansion Formula. Mechanical \& Electrical Engineering Technology, 43, 185-187. 
Submit or recommend next manuscript to SCIRP and we will provide best service for you:

Accepting pre-submission inquiries through Email, Facebook, LinkedIn, Twitter, etc. A wide selection of journals (inclusive of 9 subjects, more than 200 journals) Providing 24-hour high-quality service

User-friendly online submission system

Fair and swift peer-review system

Efficient typesetting and proofreading procedure

Display of the result of downloads and visits, as well as the number of cited articles Maximum dissemination of your research work

Submit your manuscript at: http://papersubmission.scirp.org/

Or contact eng@scirp.org 